\title{
ABOUT INTERPOLATION OF SUBSPACES OF REARRANGEMENT INVARIANT SPACES GENERATED BY RADEMACHER SYSTEM
}

\author{
SERGEY V. ASTASHKIN
}

(Received 1 August 2000 and in revised form 27 November 2000)

ABSTRACT. The Rademacher series in rearrangement invariant function spaces "close" to the space $L_{\infty}$ are considered. In terms of interpolation theory of operators, a correspondence between such spaces and spaces of coefficients generated by them is stated. It is proved that this correspondence is one-to-one. Some examples and applications are presented.

2000 Mathematics Subject Classification. Primary 46B70; Secondary 46B42, 42A55.

\section{Introduction. Let}

$$
r_{k}(t)=\operatorname{sign} \sin 2^{k-1} \pi t \quad(k=1,2, \ldots)
$$

be the Rademacher functions on the segment $[0,1]$. Define the linear operator

$$
\operatorname{Ta}(t)=\sum_{k=1}^{\infty} a_{k} r_{k}(t) \text { for } a=\left(a_{k}\right)_{k=1}^{\infty} \in l_{2} .
$$

It is well known (cf. [23, pages 340-342]) that $\mathrm{Ta}$ is an almost everywhere finite function on $[0,1]$. Moreover, from Khintchine's inequality it follows that

$$
\|T a\|_{L_{p}} \asymp\|a\|_{2} \text { for } 1 \leq p<\infty,
$$

where $\|a\|_{p}=\left(\sum_{k=1}^{\infty}\left|a_{k}\right|^{p}\right)^{1 / p}$. The symbol $\asymp$ means the existence of two-sided estimates with constants depending only on $p$. Also, it can easily be checked that

$$
\|T a\|_{L_{\infty}}=\|a\|_{1} \text {. }
$$

A more detailed information on the behaviour of Rademacher series can be obtained by treating them in the framework of general rearrangement invariant spaces.

Recall that a Banach space $X$ of measurable functions $x=x(t)$ on $[0,1]$ is said to be a rearrangement invariant space (r.i.s.) if the inequality $x^{*}(t) \leq y^{*}(t)$, for $t \in$ $[0,1]$ and $y \in X$, implies $x \in X$ and $\|x\| \leq\|y\|$. Here and in what follows $z^{*}(t)$ is the nonincreasing rearrangement of a function $|z(t)|$ with respect to the Lebesgue measure denoted by meas [10, page 83$]$.

Important examples of r.i.s.'s are Marcinkiewicz and Orlicz spaces. Let $\mathscr{P}$ denote the cone of nonnegative increasing concave functions on the semiaxis $(0, \infty)$.

If $\varphi \in \mathscr{P}$, then the Marcinkiewicz space $M(\varphi)$ consists of all measurable functions $x=x(t)$ such that

$$
\|x\|_{M(\varphi)}=\sup \left\{\frac{1}{\varphi(t)} \int_{o}^{t} x^{*}(s) d s: 0<t \leq 1\right\}<\infty .
$$


If $S(t)$ is a nonnegative convex continuous function on $[0, \infty), S(0)=0$, then the Orlicz space $L_{S}$ consists of all measurable functions $x=x(t)$ such that

$$
\|x\|_{S}=\inf \left\{u>0: \int_{0}^{1} S\left(\frac{|x(t)|}{u}\right) d t \leq 1\right\}<\infty .
$$

In particular, if $S(t)=t^{p}(1 \leq p<\infty)$, then $L_{S}=L_{p}$.

For any r.i.s. $X$ on $[0,1]$ we have $L_{\infty} \subset X \subset L_{1}\left[10\right.$, page 124]. Let $X^{0}$ denote the closure of $L_{\infty}$ in an r.i.s. $X$.

In problems discussed below, a special role is played by the Orlicz space $L_{N}$, where $N(t)=\exp \left(t^{2}\right)-1$ or, more precisely, by the space $G=L_{N}^{0}$. In [19], V. A. Rodin and E. M. Semenov proved a theorem about the equivalence of Rademacher system to the standard basis in the space $l_{2}$.

THEOREM 1.1. Suppose that $X$ is an r.i.s. Then

$$
\|T a\|_{X}=\left\|\sum_{k=1}^{\infty} a_{k} r_{k}\right\|_{X} \asymp\|a\|_{2}
$$

if and only if $X \supset G$.

By Theorem 1.1, the space $G$ is the minimal space among r.i.s.'s $X$ such that the Rademacher system is equivalent in $X$ to the standard basis of $l_{2}$.

In this paper, we consider problems related to the behaviour of Rademacher series in r.i.s.'s intermediate between $L_{\infty}$ and $G$. Here a major role is played by concepts and methods of interpolation theory of operators.

For a Banach couple $\left(X_{0}, X_{1}\right), x \in X_{0}+X_{1}$ and $t>0$, we introduce the Peetre $\mathscr{K}$ functional

$$
\mathscr{K}\left(t, x ; X_{0}, X_{1}\right)=\inf \left\{\left\|x_{0}\right\|_{X_{0}}+t\left\|x_{1}\right\|_{X_{1}}: x=x_{0}+x_{1}, x_{0} \in X_{0}, x_{1} \in X_{1}\right\} .
$$

Let $Y_{0}$ be a subspace of $X_{0}$ and $Y_{1}$ a subspace of $X_{1}$. A couple $\left(Y_{0}, Y_{1}\right)$ is called a $\mathscr{K}$-subcouple of a couple $\left(X_{0}, X_{1}\right)$ if

$$
\mathscr{K}\left(t, y ; Y_{o}, Y_{1}\right) \asymp \mathscr{K}\left(t, y ; X_{0}, X_{1}\right),
$$

with constants independent of $y \in Y_{0}+Y_{1}$ and $t>0$.

In particular, if $Y_{i}=P\left(X_{i}\right)$, where $P$ is a linear projector bounded from $X_{i}$ into itself for $i=0,1$, then $\left(Y_{0}, Y_{1}\right)$ is a $\mathcal{K}$-subcouple of $\left(X_{0}, X_{1}\right)$ (see [3] or [21, page 136]). At the same time, there are many examples of subcouples that are not $\mathscr{K}$-subcouples (see [21, page 589], [22], and Remark 3.2 of this paper).

Let $T\left(l_{1}\right)$ (respectively $T\left(l_{2}\right)$ ) denote the subspace of $L_{\infty}$ (of $G$ ) consisting of all functions of the form $x=T a$, where $T$ is given by (1.2) and $a \in l_{1}\left(\in l_{2}\right)$. From (1.4) and Theorem 1.1 it follows that

$$
\mathscr{K}\left(t, T a ; T\left(l_{1}\right), T\left(l_{2}\right)\right) \asymp \mathscr{K}\left(t, a ; l_{1}, l_{2}\right) .
$$

In spite of the fact that $T\left(l_{1}\right)$ is uncomplemented in $L_{\infty}$ (see [17] or [11, page 134]) the following assertion holds. 
THEOREM 1.2. The couple $\left(T\left(l_{1}\right), T\left(l_{2}\right)\right)$ is a $\mathscr{K}$-subcouple of the couple $\left(L_{\infty}, G\right)$. In other words (see (1.10)),

$$
\mathscr{K}\left(t, T a ; L_{\infty}, G\right) \asymp \mathscr{K}\left(t, a ; l_{1}, l_{2}\right),
$$

with constants independent of $a=\left(a_{k}\right)_{k=1}^{\infty} \in l_{2}$ and $t>0$.

We will use in the proof of Theorem 1.2 an assertion about the distribution of Rademacher sums. It was proved by S. Montgomery-Smith [13].

THEOREM 1.3. There exists a constant $A \geq 1$ such that for all $a=\left(a_{k}\right)_{k=1}^{\infty} \in l_{2}$ and $t>0$

$$
\begin{aligned}
& \operatorname{meas}\left\{s \in[0,1]: \sum_{k=1}^{\infty} a_{k} r_{k}(s)>\varphi_{a}(t)\right\} \leq \exp \left(-\frac{t^{2}}{2}\right), \\
& \operatorname{meas}\left\{s \in[0,1]: \sum_{k=1}^{\infty} a_{k} r_{k}(s)>A^{-1} \varphi_{a}(t)\right\} \geq A^{-1} \exp \left(-A t^{2}\right),
\end{aligned}
$$

where $\varphi_{a}(t)=\mathscr{K}\left(t, a ; l_{1}, l_{2}\right)$.

Now we need some definitions from interpolation theory of operators. We say that a linear operator $U$ is bounded from a Banach couple $\vec{X}=\left(X_{0}, X_{1}\right)$ into a Banach couple $\vec{Y}=\left(Y_{0}, Y_{1}\right)$ (in short, $U: \vec{X} \rightarrow \vec{Y}$ ) if $U$ is defined on $X_{0}+X_{1}$ and acts as bounded operator from $X_{i}$ into $Y_{i}$ for $i=0,1$.

Let $\vec{X}=\left(X_{0}, X_{1}\right)$ be a Banach couple. A space $X$ such that $X_{0} \cap X_{1} \subset X \subset X_{0}+X_{1}$ is called an interpolation space between $X_{0}$ and $X_{1}$ if each linear operator $U: \vec{X} \rightarrow \vec{X}$ is bounded from $X$ into itself.

To every r.i.s. $X$ assign the sequence space $F_{X}$ of Rademacher coefficients of functions of the form (1.2) from $X$ :

$$
\left\|\left(a_{k}\right)\right\|_{F_{X}}=\left\|\sum_{k=1}^{\infty} a_{k} r_{k}\right\|_{X} .
$$

Well-known properties of Rademacher functions imply that $F_{X}$ is an r.i. sequence space [19]. Furthermore, Theorem 1.3 and properties of the $\mathscr{K}$-functional show that $F_{X}$ is an interpolation space between $l_{1}$ and $l_{2}$ (see the proof of Theorem 1.2 later). For interpolation r.i.s. between $L_{\infty}$ and $G$ the correspondence $X \mapsto F_{X}$ can be defined by using the real interpolation method.

For every $p \in[1, \infty]$, we denote by $l_{p}\left(u_{k}\right), u_{k} \geq 0(k=0,1, \ldots)$ the space of all two-sided sequences of real numbers $a=\left(a_{k}\right)_{k=-\infty}^{\infty}$ such that the norm $\|a\|_{l_{p}\left(u_{k}\right)}=$ $\left\|\left(a_{k} u_{k}\right)\right\|_{p}$ is finite. Let $E$ be a Banach lattice of two-sided sequences, $\left(\min \left(1,2^{k}\right)\right)_{k=-\infty}^{\infty}$ $\in E$. If $\left(X_{0}, X_{1}\right)$ is a Banach couple, then the space of the real $\mathscr{K}$-method of interpolation $\left(X_{0}, X_{1}\right)_{E}^{\mathscr{H}}$ consists of all $x \in X_{0}+X_{1}$ such that

$$
\|x\|=\left\|\left(\mathscr{K}\left(2^{k}, x ; X_{0}, X_{1}\right)\right)_{k}\right\|_{E}<\infty .
$$

It is readily checked that the space $\left(X_{0}, X_{1}\right)_{E}^{\mathscr{F}}$ is an interpolation space between $X_{0}$ and $X_{1}$ (cf. [15, page 422]). In the special case $E=l_{p}\left(2^{-k \theta}\right)(0<\theta<1,1 \leq p \leq \infty)$ we obtain the spaces $\left(X_{0}, X_{1}\right)_{\theta, p}$ (for the detailed exposition of their properties see [4]). 
A couple $\vec{X}=\left(X_{0}, X_{1}\right)$ is said to be a $\mathscr{K}$-monotone couple if for every $x \in X_{0}+X_{1}$ and $y \in X_{0}+X_{1}$ there exists a linear operator $U: \vec{X} \rightarrow \vec{X}$ such that $y=U x$ whenever

$$
\mathscr{K}\left(t, y ; X_{0}, X_{1}\right) \leq \mathscr{K}\left(t, x ; X_{0}, X_{1}\right) \quad \forall t>0 .
$$

As it is well known (cf. [15, page 482]), any interpolation space $X$ with respect to a $\mathscr{K}$-monotone couple $\left(X_{0}, X_{1}\right)$ is described by the real $\mathscr{K}$-method. It means that for some $E$

$$
X=\left(X_{0}, X_{1}\right)_{E}^{\mathscr{K}}
$$

In particular, by the Sparr theorem [20] the couple $\left(l_{1}, l_{2}\right)$ is a $\mathscr{K}$-monotone couple. Therefore, if $F$ is an interpolation space between $l_{1}$ and $l_{2}$, then there exists $E$ such that

$$
F=\left(l_{1}, l_{2}\right)_{E}^{\mathscr{K}}
$$

Hence Theorem 1.2 allows to find an r.i.s. that contains Rademacher series with coefficients belonging to an arbitrary interpolation space between $l_{1}$ and $l_{2}$. In [19], the similar result was obtained for sequence spaces satisfying more restrictive conditions (see Remark 3.3).

THEOREM 1.4. Let $F$ be an interpolation sequence space between $l_{1}$ and $l_{2}$ and $F=$ $\left(l_{1}, l_{2}\right)_{E}^{\mathscr{H}}$. Then for the r.i.s. $X=\left(L_{\infty}, G\right)_{E}^{\mathscr{H}_{\mathrm{C}}}$ we have

$$
\left\|\sum_{k=1}^{\infty} a_{k} r_{k}\right\|_{X} \asymp\|a\|_{F}
$$

with constants independent of $a=\left(a_{k}\right)_{k=1}^{\infty}$.

Combining Theorem 1.4 with the above remarks, we get the following assertion. If $F$ is a sequence space, then

$$
\left\|\left(a_{k}\right)\right\|_{F} \asymp\left\|\sum_{k=1}^{\infty} a_{k} r_{k}\right\|_{X} \text { for some r.i.s. } X
$$

if and only if $F$ is an interpolation space between $l_{1}$ and $l_{2}$.

The last result shows that the restriction of the correspondence (1.13) to interpolation r.i.s. between $L_{\infty}$ and $G$ is bijective.

THEOREM 1.5. Let r.i.s.'s $X_{0}$ and $X_{1}$ be two interpolation spaces between $L_{\infty}$ and $G$. If

$$
\left\|\sum_{k=1}^{\infty} a_{k} r_{k}\right\|_{X_{0}} \asymp\left\|\sum_{k=1}^{\infty} a_{k} r_{k}\right\|_{X_{1}},
$$

then $X_{0}=X_{1}$ and the norms of $X_{0}$ and $X_{1}$ are equivalent.

In $[16,19]$, the similar results were obtained by additional conditions with respect to spaces $X_{0}$ and $X_{1}$. 


\section{Proofs}

Proof OF TheOrem 1.2. It is known [10, page 164] that the $\mathscr{K}$-functional of a couple of Marcinkiewicz spaces is given by the formula

$$
\mathscr{K}\left(t, x ; M\left(\varphi_{0}\right), M\left(\varphi_{1}\right)\right)=\sup _{0<u \leq 1} \frac{\int_{0}^{u} x^{*}(s) d s}{\max \left(\varphi_{0}(u), \varphi_{1}(u) / t\right)} .
$$

If $N(t)=\exp \left(t^{2}\right)-1$, then the Orlicz space $L_{N}$ coincides with the Marcinkiewicz space $M\left(\varphi_{1}\right)$, where $\varphi_{1}(u)=u \log _{2}^{1 / 2}(2 / u)$ [12]. In addition, $L_{\infty}=M\left(\varphi_{0}\right)$, where $\varphi_{0}(u)=u$. Therefore,

$$
\mathscr{K}\left(t, x ; L_{\infty}, G\right)=\sup _{0<u \leq 1}\left\{\frac{1}{u} \int_{0}^{u} x^{*}(s) d s \min \left(1, t \log _{2}^{-1 / 2}\left(\frac{2}{u}\right)\right)\right\} \quad \text { for } x \in G .
$$

Since $x^{*}(u) \leq 1 / u \int_{0}^{u} x^{*}(s) d s$, then from (2.2) it follows that

$$
\mathscr{K}\left(t, x ; L_{\infty}, G\right) \geq \sup _{k=0,1, \ldots}\left\{x^{*}\left(2^{-k}\right) \min \left(1, t(k+1)^{-1 / 2}\right)\right\} .
$$

Hence,

$$
\mathscr{K}\left(t, x ; L_{\infty}, G\right) \geq x^{*}\left(2^{-k_{t}}\right) \text { for } t \geq 1,
$$

where $k_{t}=\left[t^{2}\right]-1([z]$ is the integral part of a number $z)$.

Now let $a=\left(a_{k}\right)_{k=1}^{\infty} \in l_{2}$ and $x(t)=T a(t)=\sum_{k=1}^{\infty} a_{k} r_{k}(t)$. By the Holmstedt formula [7],

$$
\varphi_{a}(t) \leq \sum_{k=1}^{\left[t^{2}\right]} a_{k}^{*}+t\left\{\sum_{k=\left[t^{2}\right]+1}^{\infty}\left(a_{k}^{*}\right)^{2}\right\}^{1 / 2} \leq B \varphi_{a}(t),
$$

where $\varphi_{a}(t)=\mathscr{K}\left(t, a ; l_{1}, l_{2}\right),\left(a_{k}^{*}\right)_{k=1}^{\infty}$ is a nonincreasing rearrangement of the sequence $\left(\left|a_{k}\right|\right)_{k=1}^{\infty}$, and $B>0$ is a constant independent of $a=\left(a_{k}\right)_{k=1}^{\infty}$ and $t>0$.

Assume, at first, that $a \notin l_{1}$. Then inequality (2.5) shows that

$$
\lim _{t \rightarrow 0+} \varphi_{a}(t)=0, \quad \lim _{t \rightarrow \infty} \varphi_{a}(t)=\infty .
$$

The function $\varphi_{a}$ belongs to the class $\mathscr{P}[4$, page 55]. Therefore it maps the semiaxis $(0, \infty)$ onto $(0, \infty)$ one-to-one, and there exists the inverse function $\varphi_{a}^{-1}$. By Theorem 1.3, we have

$$
n_{|x|}(\tau)=\operatorname{meas}\{s \in[0,1]:|x(s)|>\tau\} \geq \psi(\tau) \text { for } \tau>0,
$$

where $\psi(\tau)=A^{-1} \exp \left\{-A\left[\varphi_{a}^{-1}(\tau A)\right]^{2}\right\}$. Passing to rearrangements we obtain

$$
x^{*}(s) \geq \psi^{-1}(s) \text { for } 0<s<A^{-1} .
$$

Obviously, by condition $t \geq C_{1}=C_{1}(A)=\sqrt{2 \log _{2}(2 A)}$, it holds

$$
2^{-k_{t} / 2}<A^{-1} \text { for } k_{t}=\left[t^{2}\right]-1 .
$$

Hence (2.4) and (2.8) imply

$$
\mathscr{K}\left(t, x ; L_{\infty}, G\right) \geq \psi^{-1}\left(2^{-k_{t}}\right) .
$$


Combining the definition of the function $\psi$ with (2.9), we obtain

$$
\begin{aligned}
\psi^{-1}\left(2^{-k_{t}}\right) & =A^{-1} \varphi_{a}\left(A^{-1 / 2} \ln ^{1 / 2}\left(A^{-1} 2^{k_{t}}\right)\right) \geq A^{-1} \varphi_{a}\left(\sqrt{\frac{k_{t} \ln 2}{2 A}}\right) \\
& \geq A^{-3 / 2} \sqrt{\frac{\ln 2}{2}} \varphi_{a}\left(\sqrt{k_{t}}\right) \geq A^{-3 / 2} \sqrt{\frac{\ln 2}{2}} t^{-1} \sqrt{k_{t}} \varphi_{a}(t) .
\end{aligned}
$$

From the inequality $t \geq C_{1} \geq \sqrt{2}$ it follows that

$$
\frac{\sqrt{k_{t}}}{t} \geq \frac{\sqrt{\left[t^{2}\right]-1}}{\sqrt{\left[t^{2}\right]+1}} \geq 3^{-1 / 2}
$$

Therefore, by (2.10), we have

$$
\mathscr{K}\left(t, x ; L_{\infty}, G\right) \geq C_{2} \varphi_{a}(t) \text { for } t \geq C_{1},
$$

where $C_{2}=C_{2}(A)=\sqrt{\ln 2 / 6} A^{-3 / 2}$.

If now $t \geq 1$, then the concavity of the $\mathscr{K}$-functional and the previous inequality yield

$$
\mathscr{K}\left(t, x ; L_{\infty}, G\right) \geq C_{1}^{-1} \mathscr{K}\left(t C_{1}, x ; L_{\infty}, G\right) \geq \frac{C_{2}}{C_{1}} \varphi_{a}\left(C_{1} t\right) \geq \frac{C_{2}}{C_{1}} \varphi_{a}(t) .
$$

Using the inequalities $\|a\|_{2} \leq\|a\|_{1}\left(a \in l_{1}\right)$ and $\|x\|_{G} \leq\|x\|_{\infty}\left(x \in L_{\infty}\right)$, the definition of the $\mathscr{K}$-functional, and Theorem 1.1, we obtain

$$
\mathscr{K}\left(t, x ; L_{\infty}, G\right)=t\|x\|_{G} \geq C_{3} t\|a\|_{2}=C_{3} \varphi_{a}(t) \quad \text { for } 0<t \leq 1 .
$$

Thus,

$$
\mathscr{K}\left(t, a ; l_{1}, l_{2}\right) \leq C \mathscr{K}\left(t, T a ; L_{\infty}, G\right),
$$

if $C=\max \left(C_{3}^{-1}, C_{1} / C_{2}\right)$.

Suppose now $a \in l_{1}$. By (2.5), without loss of generality, we can assume that the function $\varphi_{a}$ maps the semiaxis $(0, \infty)$ injectively onto the interval $\left(0,\|a\|_{1}\right)$. Hence we can define the mappings $\varphi_{a}^{-1}:\left(0,\|a\|_{1}\right) \rightarrow(0, \infty), \psi:\left(0, A^{-1}\|a\|_{1}\right) \rightarrow\left(0, A^{-1}\right)$, and $\psi^{-1}:\left(0, A^{-1}\right) \rightarrow\left(0, A^{-1}\|a\|_{1}\right)$. Arguing as above, we get inequality (2.16).

The opposite inequality follows from Theorem 1.1 and relation (1.4). Indeed,

$$
\begin{aligned}
\mathscr{K}\left(t, T a ; L_{\infty}, G\right) & \leq \inf \left\{\left\|T a^{0}\right\|_{\infty}+t\left\|T a^{1}\right\|_{G}: a=a^{0}+a^{1}, a^{0} \in l_{1}, a^{1} \in l_{2}\right\} \\
& \leq D \mathscr{K}\left(t, a ; l_{1}, l_{2}\right) .
\end{aligned}
$$

Proof OF TheOrem 1.4. It is sufficient to use Theorem 1.2 and the definition of the real $\mathscr{K}$-method of interpolation.

For the proof of Theorem 1.5 we need some definitions and auxiliary assertions. These results are also of some independent interest.

Let $f(t)$ be a function defined on the interval $(0, l)$, where $l=1$ or $l=\infty$. Then the dilation function of $f$ is defined as follows:

$$
\mathcal{M}_{f}(t)=\sup \left\{\frac{f(s t)}{f(s)}: s, s t \in(0, l)\right\}, \quad \text { if } t \in(0, l) .
$$


Since this function is semimultiplicative, then there exist numbers

$$
\gamma_{f}=\lim _{t \rightarrow 0+} \frac{\ln M_{f}(t)}{\ln t}, \quad \delta_{f}=\lim _{t \rightarrow \infty} \frac{\ln M_{f}(t)}{\ln t} .
$$

A Banach couple $\vec{X}=\left(X_{0}, X_{1}\right)$ is called a partial retract of a couple $\vec{Y}=\left(Y_{0}, Y_{1}\right)$ if each element $x \in X_{0}+X_{1}$ is orbitally equivalent to some element $y \in Y_{0}+Y_{1}$. The last means that there exist linear operators $U: \vec{X} \rightarrow \vec{Y}$ and $V: \vec{Y} \rightarrow \vec{X}$ such that $U x=y$ and $V y=x$.

Proposition 2.1. Suppose that $M(\varphi)$ is a Marcinkiewicz space on $[0,1]$. If $\gamma_{\varphi}>0$, then $\vec{X}=\left(L_{\infty}, M(\varphi)\right)$ is a $\mathscr{K}$-monotone couple.

Proof. It is sufficient to show that the couple $\vec{X}$ is a partial retract of the couple $\vec{Y}=\left(L_{\infty}, L_{\infty}(\bar{\varphi})\right)$, where

$$
\|x\|_{L_{\infty}(\tilde{\varphi})}=\sup _{0<t \leq 1} \tilde{\varphi}(t)|x(t)|, \quad \tilde{\varphi}(t)=\frac{t}{\varphi}(t) .
$$

Indeed, a partial retract of a $\mathscr{K}$-monotone couple is a $\mathscr{K}$-monotone couple [15, page 420], and by the Sparr theorem [20] $\vec{Y}$ is a $\mathscr{K}$-monotone couple.

First note that the inclusion $L_{\infty} \subset M(\varphi)$ implies $L_{\infty}+M(\varphi)=M(\varphi)$. So, let $x \in$ $M(\varphi)$. Without loss of generality [10, page 87], assume that $x(t)=x^{*}(t)$. Define the operator

$$
U_{1} y(t)=\sum_{k=1}^{\infty} 2^{k} \int_{0}^{2^{-k}} y(s) d s \chi_{\left(2^{-k}, 2^{-k+1}\right]}(t) \text { for } y \in M(\varphi) .
$$

Clearly, $U_{1}$ maps $L_{\infty}$ into itself. In addition, the concavity of the function $\varphi$ and properties of the nonincreasing rearrangement imply

$$
\left\|U_{1} y\right\|_{L_{\infty}(\tilde{\varphi})} \leq 2 \sup _{k=1,2, \ldots}\left(\varphi\left(2^{-k+1}\right)\right)^{-1} \int_{0}^{2^{-k}} y^{*}(s) d s \leq 2\|y\|_{M(\varphi)} .
$$

Hence $U_{1}: \vec{X} \rightarrow \vec{Y}$. Since $x(t)$ is nonincreasing, then $U_{1} x(t) \geq x(t)$. Therefore the linear operator

$$
U y(t)=\frac{x(t)}{U_{1} x(t)} U_{1} y(t)
$$

is bounded from the couple $\vec{X}$ into the couple $\vec{Y}$. In addition, $U x(t)=x(t)$.

Take for $V$ the identity mapping, that is, $V y(t)=y(t)$. Since $\gamma_{f}>0$, then, by [10, page 156], we have

$$
\|V y\|_{M(\varphi)} \leq C \sup _{0<t \leq 1} \tilde{\varphi}(t) y^{*}(t) \leq C \sup _{0<t \leq 1} \tilde{\varphi}(t)|y(t)|=C\|y\|_{L_{\infty}(\tilde{\varphi})} .
$$

Therefore $V: \vec{Y} \rightarrow \vec{X}$ and $V x=x$.

Thus an arbitrary element $x \in M(\varphi)$ is orbitally equivalent to itself as to element of the space $L_{\infty}+L_{\infty}(\tilde{\varphi})$. This completes the proof. 
COROLLARY 2.2. If $\gamma_{\varphi}>0$, then $\left(L_{\infty}, M(\varphi)^{0}\right)$ is a $\mathscr{K}$-monotone couple.

Proof. Assume that $x$ and $y$ belong to the space $M(\varphi)^{0}$ and

$$
\mathscr{K}\left(t, y ; L_{\infty}, M(\varphi)^{0}\right) \leq \mathscr{K}\left(t, x ; L_{\infty}, M(\varphi)^{0}\right) \text { for } t>0 .
$$

If $z \in M(\varphi)^{0}$, then

$$
\mathscr{K}\left(t, z ; L_{\infty}, M(\varphi)^{0}\right)=\mathscr{K}\left(t, z ; L_{\infty}, M(\varphi)\right)
$$

Therefore,

$$
\mathscr{K}\left(t, y ; L_{\infty}, M(\varphi)\right) \leq \mathscr{K}\left(t, x ; L_{\infty}, M(\varphi)\right) \text { for } t>0 .
$$

Hence, by Proposition 2.1, there exists an operator $T:\left(L_{\infty}, M(\varphi)\right) \rightarrow\left(L_{\infty}, M(\varphi)\right)$ such that $y=T x$. It is readily seen that $M(\varphi)^{0}$ is an interpolation space of the couple $\left(L_{\infty}, M(\varphi)\right)$. Therefore $T:\left(L_{\infty}, M(\varphi)^{0}\right) \rightarrow\left(L_{\infty}, M(\varphi)^{0}\right)$.

We define now two subcones of the cone $\mathscr{P}$. Denote by $\mathscr{P}_{0}$ the set of all functions $f \in \mathscr{P}$ such that $\lim _{t \rightarrow 0+} f(t)=\lim _{t \rightarrow \infty} f(t) / t=0$. If $f \in \mathscr{P}$, then $0 \leq \gamma_{f} \leq \delta_{f} \leq 1[10$, page 76]. Let $\mathscr{P}^{+-}$be the set of all $f \in \mathscr{P}$ such that $0<\gamma_{f} \leq \delta_{f}<1$. It is obvious that $\mathscr{P}^{+-} \subset \mathscr{P}_{0}$.

A couple $\left(X_{0}, X_{1}\right)$ is called a $\mathscr{K}_{0}$-complete couple if for any function $f \in \mathscr{P}_{0}$ there exists an element $x \in X_{0}+X_{1}$ such that

$$
\mathscr{K}\left(t, x ; X_{0}, X_{1}\right) \asymp f(t) .
$$

In other words, the set $\mathscr{K}\left(X_{0}+X_{1}\right)$ of all $\mathscr{K}$-functionals of a $\mathscr{K}_{0}$-complete couple $\left(X_{0}, X_{1}\right)$ contains, up to equivalence, the whole of the subcone $\mathscr{P}_{0}$.

Proposition 2.3. The Banach couple $\left(L_{1}(0, \infty), L_{2}(0, \infty)\right)$ is a $\mathscr{K}_{0}$-complete couple.

Proof. By the Holmstedt formula for functional spaces [7],

$$
\mathscr{K}\left(t, x, L_{1}, L_{2}\right) \asymp \max \left\{\int_{0}^{t^{2}} x^{*}(s) d s, t\left[\int_{t^{2}}^{\infty}\left(x^{*}(s)\right)^{2} d s\right]^{1 / 2}\right\} .
$$

If $f \in \mathscr{P}_{0}$, then $g(t)=f\left(t^{1 / 2}\right)$ belongs to $\mathscr{P}_{0}$. We denote $x(t)=g^{\prime}(t)$. Then $x(t)=$ $x^{*}(t)$ and

$$
\int_{0}^{t} x(s) d s=g(t)
$$

Assume that $f \in \mathscr{P}^{+-}$. If $\delta_{f}<1$, then there exists $\varepsilon>0$ such that for some $C>0$

$$
G(s)=f\left(s^{1 / 2}\right) \leq C\left(\sqrt{\frac{s}{t}}\right)^{1-\varepsilon} f\left(t^{1 / 2}\right), \quad \text { if } s \geq t .
$$

Since $g \in \mathscr{P}_{0}$, then $g^{\prime}(t) \leq g(t) / t$. Therefore for $t>0$

$$
\int_{t}^{\infty}(x(s))^{2} d s \leq \int_{t}^{\infty} \frac{g^{2}(s)}{s^{2}} d s \leq C^{2} t^{\varepsilon-1}\left(f\left(t^{1 / 2}\right)\right)^{2} \int_{t}^{\infty} s^{-1-\varepsilon} d s=C^{2} \varepsilon t^{-1}(g(t))^{2} .
$$

Combining this with (2.29) and (2.30), we obtain

$$
\mathscr{K}\left(t, x ; L_{1}, L_{2}\right) \asymp g\left(t^{2}\right)=f(t) .
$$


Thus $\mathscr{K}\left(L_{1}+L_{2}\right) \supset \mathscr{P}^{+-}$. Hence, in particular, the intersection $\mathscr{K}\left(X_{0}+X_{1}\right) \cap \mathscr{P}^{+-}$is not empty. Therefore, by [6, Theorem 4.5.7], $\left(L_{1}, L_{2}\right)$ is a $\mathscr{K}_{0}$-complete Banach couple. This completes the proof.

Let $\mathscr{K}\left(l_{1}+l_{2}\right)$ be the set of all $\mathscr{K}$-functionals corresponding to the couple $\left(l_{1}, l_{2}\right)$. By $\mathscr{F}$ we denote the set of all functions $f \in \mathscr{P}$ such that

$$
f(t)=f(1) t \quad \text { for } 0<t \leq 1, \quad \lim _{t \rightarrow \infty} \frac{f(t)}{t}=0 .
$$

COROLlary 2.4. Up to equivalence,

$$
\mathscr{K}\left(l_{1}+l_{2}\right) \supset \mathscr{F} .
$$

Proof. It is well known (cf. [4, page 142]) that for $x \in L_{1}(0, \infty)+L_{\infty}(0, \infty)$ and $u>0$

$$
\mathscr{K}\left(u, x ; L_{1}, L_{\infty}\right)=\int_{0}^{u} x^{*}(s) d s .
$$

In addition,

$$
L_{1}=\left(L_{1}, L_{\infty}\right)_{l_{\infty}}^{\mathscr{K}}, \quad L_{2}=\left(L_{1}, L_{\infty}\right)_{l_{2}\left(2^{-k / 2}\right)}^{\mathscr{K}} .
$$

The spaces $l_{\infty}$ and $l_{2}\left(2^{-k / 2}\right)$ are interpolation spaces with respect to the couple $\left(l_{\infty}, l_{\infty}\left(2^{-k}\right)\right)$ [4]. Therefore, by the reiteration theorem (see [5] or [14]),

$$
\mathscr{K}\left(t, x ; L_{1}, L_{2}\right) \asymp \mathscr{K}\left(t, \mathscr{K}\left(\cdot, x ; L_{1}, L_{\infty}\right) ; l_{\infty}, l_{2}\left(2^{-k / 2}\right)\right) \text { for } x \in L_{1}+L_{2} .
$$

Introduce the average operator:

$$
Q x(t)=\sum_{k=1}^{\infty} \int_{k-1}^{k} x(s) d s \chi_{(k-1, k]}(t), \quad \text { if } t>0 .
$$

From (2.36) it follows that

$$
\mathscr{K}\left(t, Q x^{*} ; L_{1}, L_{\infty}\right)=\mathscr{K}\left(t, x ; L_{1}, L_{\infty}\right)
$$

for all positive integers $t$. Both functions in (2.40) are concave. Therefore,

$$
\mathscr{K}\left(t, Q x^{*} ; L_{1}, L_{\infty}\right) \asymp \mathscr{K}\left(t, x ; L_{1} \cdot L_{\infty}\right) \quad \forall t \geq 1 .
$$

Hence (2.38) yields

$$
\mathscr{K}\left(t, Q x^{*} ; L_{1}, L_{2}\right) \asymp \mathscr{K}\left(t, x ; L_{1}, L_{2}\right), \quad \text { if } t \geq 1 .
$$

Now let $f \in \mathscr{F}$. Since $\mathscr{F}_{\mathcal{C}} \subset \mathscr{P}_{0}$, then, by Proposition 2.3, there exists a function $x \in$ $L_{1}(0, \infty)+L_{2}(0, \infty)$ such that

$$
\mathscr{K}\left(t, x ; L_{1}, L_{2}\right) \asymp f(t) .
$$

Clearly, the operator $Q$ is a projector in the spaces $L_{1}$ and $L_{2}$ with norm 1 . Moreover, $Q\left(L_{1}\right)=l_{1}$ and $Q\left(L_{2}\right)=l_{2}$. Hence, by the theorem about complemented subcouples 
mentioned in Section 1 (see [3] or [21, page 136]),

$$
\mathscr{K}\left(t, Q x^{*} ; L_{1}, L_{2}\right) \asymp \mathscr{K}\left(t, a ; l_{1}, l_{2}\right) \text { for } t>0 \text {, }
$$

where $a=\left(\int_{k-1}^{k} x^{*}(s) d s\right)_{k=1}^{\infty}$.

Thus (2.42) and (2.43) imply

$$
\mathscr{K}\left(t, a ; l_{1}, l_{2}\right) \asymp f(t) \quad \text { for } t \geq 1 .
$$

The last relation also holds if $0<t \leq 1$. Indeed, in this case

$$
\mathscr{K}\left(t, a ; l_{1}, l_{2}\right)=t\|a\|_{2}=t \mathscr{K}\left(1, a ; l_{1}, l_{2}\right) \asymp t f(1)=f(t) .
$$

This completes the proof.

Proof OF TheOrem 1.5. As it was already mentioned in the proof of Theorem 1.2, the Orlicz space $L_{N}, N(t)=\exp \left(t^{2}\right)-1$, coincides with the Marcinkiewicz space $M\left(\varphi_{1}\right)$, for $\varphi_{1}(u)=u \log _{2}^{1 / 2}(2 / u)$. Since $\gamma_{\varphi_{1}}=1$, then Corollary 2.2 implies that the couple $\left(L_{\infty}, G\right)$ is a $\mathscr{K}$-monotone couple. Hence,

$$
X_{0}=\left(l_{\infty}, G\right)_{E_{0}}^{\mathscr{K}}, \quad X_{1}=\left(l_{\infty}, G\right)_{E_{1}}^{\mathscr{K}},
$$

for some parameters of the real $\mathscr{K}$-method of interpolation $E_{0}$ and $E_{1}$. By Theorem 1.4,

$$
\left\|\sum_{k=1}^{\infty} a_{k} r_{k}\right\|_{X_{i}} \asymp\left\|\left(a_{k}\right)\right\|_{F_{i}},
$$

where $F_{i}=\left(l_{1}, l_{2}\right)_{E_{i}}^{\mathscr{H}_{C}}(i=0,1)$. So

$$
\left(l_{1}, l_{2}\right)_{E_{0}}^{\mathscr{K}}=\left(l_{1}, l_{2}\right)_{E_{1}}^{\mathscr{H}} .
$$

Equation (2.49) means that the norms of spaces $E_{0}$ and $E_{1}$ are equivalent on the set $\mathscr{K}\left(l_{1}+l_{2}\right)$. It is readily to check that this set coincides, up to the equivalence, with the set $\mathscr{K}\left(L_{\infty}+G\right)$ of all $\mathscr{K}$-functionals corresponding to the couple $\left(L_{\infty}, G\right)$. More precisely,

$$
\mathscr{K}\left(l_{1}+l_{2}\right)=\mathscr{K}\left(L_{\infty}+G\right)=\mathscr{F} .
$$

In fact, by Theorem 1.2 and Corollary 2.2, $\mathscr{F} \subset \mathscr{K}\left(l_{1}+l_{2}\right) \subset \mathscr{K}\left(L_{\infty}+G\right)$. On the other hand, since $L_{\infty} \subset G$ with the constant 1 and $L_{\infty}$ is dense in $G$, then $\mathscr{K}\left(L_{\infty}+G\right) \subset \mathscr{F}$ [15, page 386].

Now let $x \in X_{0}$. By (2.47), we have $\left(\mathscr{K}\left(2^{k}, x ; L_{\infty}, G\right)\right)_{k} \in X_{0}$. Using (2.50), we can find $a \in l_{2}$ such that

$$
\mathscr{K}\left(2^{k}, a ; l_{1}, l_{2}\right) \asymp \mathscr{K}\left(2^{k}, x ; L_{\infty}, G\right)
$$

for all positive integers $k$. Since a parameter of $\mathscr{K}$-method is a Banach lattice, then this implies $\left(\mathscr{K}\left(2^{k}, a ; l_{1}, l_{2}\right)\right)_{k} \in E_{0}$. Therefore, by $(2.49),\left(\mathscr{K}\left(2^{k}, a ; l_{1}, l_{2}\right)\right)_{k} \in E_{1}$, that is, $\left(\mathscr{K}\left(2^{k}, x ; L_{\infty}, G\right)\right)_{k} \in E_{1}$ or $x \in X_{1}$. Thus $X_{0} \subset X_{1}$. Arguing as above, we obtain the converse inclusion, and $X_{0}=X_{1}$ as sets. Since $X_{0}$ and $X_{1}$ are Banach lattices, then their norms are equivalent. This completes the proof. 


\section{Final remarks and examples}

REMARK 3.1. Combining Theorems 1.2, 1.4, and 1.5 with results obtained in [8], we can prove similar assertions for lacunary trigonometric series. Moreover, taking into account the main result of [1], we can extend Theorems 1.2, 1.4, and 1.5 to Sidon systems of characters of a compact abelian group.

REMARK 3.2. In Theorem 1.2, we cannot replace the space $G$ by $L_{q}$ with some $q<\infty$. Indeed, suppose that the couple $\left(T\left(l_{1}\right), T\left(l_{2}\right)\right)$ is a $\mathscr{K}$-subcouple of the couple $\left(L_{\infty}, L_{q}\right)$, that is,

$$
\mathscr{K}\left(t, a ; l_{1}, l_{2}\right) \asymp \mathscr{K}\left(t, T a ; L_{\infty}, L_{q}\right) .
$$

Let $E=l_{p}\left(2^{-\theta k}\right)$, where $0<\theta<1$ and $p=q / \theta$. Applying the $\mathscr{K}$-method of interpolation $(\cdot, \cdot)_{E}^{\mathscr{K}}$ to the couples $\left(l_{1}, l_{2}\right)$ and $\left(L_{\infty}, L_{q}\right)$, we obtain

$$
\|T a\|_{p} \asymp\|a\|_{r, p}=\left\{\sum_{k=1}^{\infty}\left(a_{k}^{*}\right)^{p} k^{p / r-1}\right\}^{1 / p} .
$$

Since $r=2 /(2-\theta)<2$ [4, page 142], then this contradicts with (1.3).

REMARK 3.3. Clearly, a partial retract of a couple $\vec{Y}=\left(Y_{0}, Y_{1}\right)$ is a $\mathscr{K}$-subcouple of $\vec{Y}$. The opposite assertion is not true, in general (nevertheless, some interesting examples of $\mathscr{K}$-subcouples and partial retracts simultaneously are given in [9]). Indeed, by Theorem 1.2 , the subcouple $\left(l_{1}, l_{2}\right)$ is a $\mathscr{K}$-subcouple of the couple $\left(L_{\infty}, G\right)$. Assume that $\left(l_{1}, l_{2}\right)$ is a partial retract of this couple. Then (see the proof of Proposition 2.1) $\left(l_{1}, l_{2}\right)$ is a partial retract of the couple $\left(L_{\infty}, L_{\infty}\left(\log _{2}^{-1 / 2}(2 / t)\right)\right)$, as well. Therefore, by Lemma 1 from [2] and [4, page 142] it follows that

$$
\left[l_{1}, l_{2}\right]_{\theta}=\left(l_{1}, l_{2}\right)_{\theta, \infty}=l_{p, \infty},
$$

where $\left[l_{1}, l_{2}\right]_{\theta}$ is the space of the complex method of interpolation [4], $0<\theta<1$, and $p=2 /(2-\theta)$. On the other hand, it is well known [4, page 139] that

$$
\left[l_{1}, l_{2}\right]_{\theta}=l_{p} \text { for } p=\frac{2}{2-\theta} .
$$

This contradiction shows that the couple $\left(l_{1}, l_{2}\right)$ is not a partial retract of the couple $\left(L_{\infty}, G\right)$.

Using Theorem 1.4, we can find coordinate sequence spaces of coefficients of Rademacher series belonging to certain r.i.s.'s.

EXAMPLE 3.4. Let $X$ be the Marcinkiewicz space $M(\varphi)$, where $\varphi(t)=t \log _{2} \log _{2}(16 / t)$, $0<t \leq 1$. Show that

$$
\left\|\sum_{k=1}^{\infty} a_{k} r_{k}\right\|_{M(\varphi)} \asymp\|a\|_{l_{1}(\log )},
$$

where $l_{1}(\log )$ is the space of all sequences $a=\left(a_{k}\right)_{k=1}^{\infty}$ such that the norm

$$
\|a\|_{l_{1}(\log )}=\sup _{k=1,2, \ldots} \log _{2}^{-1}(2 k) \sum_{i=1}^{k} a_{i}^{*}
$$


is finite. Taking into account Theorem 1.4, it is sufficient to check that

$$
\begin{aligned}
& \left(l_{1}, l_{2}\right)_{F}^{\mathscr{K}}=l_{1}(\log ), \\
& \left(l_{\infty}, G\right)_{F}^{\mathscr{K}}=M(\varphi),
\end{aligned}
$$

for some parameter $F$ of the $\mathscr{K}$-method of interpolation. More precisely, we will prove that (3.7) and (3.8) are true for $F=l_{\infty}\left(u_{k}\right)$, where $u_{k}=1 /(k+1)(k \geq 0)$ and $u_{k}=1$ $(k<0)$.

By the Holmstedt formula (2.5),

$$
\varphi_{a}\left(2^{k}\right) \leq \sum_{i=1}^{2^{2 k}} a_{i}^{*}+2^{k}\left[\sum_{i=2^{2 k}+1}^{\infty}\left(a_{i}^{*}\right)^{2}\right]^{1 / 2} \leq B \varphi_{a}\left(2^{k}\right) \text { for } k=0,1,2, \ldots,
$$

where, as before, $\varphi_{a}(t)=\mathscr{K}\left(t, a ; l_{1}, l_{2}\right)$. Without loss of generality, assume that $a_{i}=$ $a_{i}^{*}$. If $\|a\|_{l_{1}(\log )}=R<\infty$, then by (3.6),

$$
\sum_{i=1}^{2 k} a_{i}^{*} \leq 2 R(k+1)
$$

In particular, this implies $a_{22 k} \leq 2^{-2 k+1} R(k+1)$, for nonnegative integer $k$. Using (3.10), we obtain

$$
\begin{aligned}
\sum_{i=2^{2 k}+1}^{\infty} a_{i}^{2} & =\sum_{j=k}^{\infty} \sum_{i=2^{2 j}+1}^{2^{2(j+1)}} a_{i}^{2} \leq 3 \sum_{j=k}^{\infty} 2^{2 j} a_{2^{2 j}}^{2} \leq 12 R^{2} \sum_{j=k}^{\infty} 2^{-2 j}(j+1)^{2} \\
& \leq 192 R^{2} \int_{k+1}^{\infty} x^{2} 2^{-2 x} d x \leq 144 R^{2}(k+1)^{2} 2^{-2 k}
\end{aligned}
$$

Hence the second term in (3.9) does not exceed $12 R(k+1)$. Therefore, if $E=\left(l_{1}, l_{2}\right)_{F}^{\mathscr{F}}$, then (3.10) implies

$$
\|a\|_{E}=\sup _{k=0,1, \ldots} \frac{\varphi_{a}\left(2^{k}\right)}{k+1} \leq 14\|a\|_{l_{1}(\log )} .
$$

Conversely, if $2^{2 j}+1 \leq k \leq 2^{2(j+1)}$ for some $j=0,1,2, \ldots$, then from (3.9) it follows that

$$
\sum_{i=1}^{k} a_{i} \leq B \varphi_{a}\left(2^{j+1}\right) \leq \sum_{i=1}^{2^{2(j+1)}} a_{i} \leq B\|a\|_{E}(j+2) \leq 2 B \log _{2}(2 k)\|a\|_{E} .
$$

Therefore, $\|a\|_{l_{1}(\log )} \leq 2 B\|a\|_{E}$ and (3.7) is proved.

We pass now to function spaces. At first, we introduce one more interpolation method which is, actually, a special case of the real method of interpolation. For a function $\varphi \in \mathscr{P}$ and an arbitrary Banach couple $\left(X_{0}, X_{1}\right)$ define generalized Marcinkiewicz space as follows:

$$
M_{\varphi}\left(X_{0}, X_{1}\right)=\left\{x \in X_{0}+X_{1}: \sup _{t>0} \frac{\mathscr{K}\left(t, x ; X_{0}, X_{1}\right)}{\varphi(t)}<\infty\right\} .
$$


Let $\varphi_{0}(t)=\min (1, t), \varphi_{1}(t)=\min \left(1, t \log _{2}^{1 / 2}[\max (2,2 / t)]\right)$, and $N(t)=\exp \left(t^{2}\right)-1$, as before. By equation (2.36), we have

$$
L_{\infty}=M_{\varphi_{0}}\left(L_{1}, L_{\infty}\right), \quad L_{N}=M_{\varphi_{1}}\left(L_{1}, L_{\infty}\right),
$$

(here $L_{\infty}$ and $L_{N}$ are functional spaces on the segment [0,1]). In addition, using similar notation, it is easy to check that

$$
\left(X_{0}, X_{1}\right)_{F}^{\mathscr{K}}=M_{\rho}\left(X_{0}, X_{1}\right)
$$

for an arbitrary Banach couple $\left(X_{0}, X_{1}\right)$ and $\rho(t)=\log _{2}(4+t)$. Hence, by the reiteration theorem for generalized Marcinkiewicz spaces [15, page 428], we obtain

$$
\left(L_{\infty}, L_{N}\right)_{F}^{\mathscr{K}}=M_{\rho}\left(M_{\varphi_{0}}\left(L_{1}, L_{\infty}\right), M_{\varphi_{1}}\left(L_{1}, L_{\infty}\right)\right)=M_{\varphi_{\rho}}\left(L_{1}, L_{\infty}\right)=M\left(\varphi_{\rho}\right),
$$

where $\varphi_{\rho}(t)=\varphi_{0}(t) \rho\left(\varphi_{1}(t) / \varphi_{0}(t)\right)$. A simple calculation gives $\varphi_{\rho}(t) \asymp \varphi(t)$, if $t>0$. Thus,

$$
\left(L_{\infty}, L_{N}\right)_{F}^{\mathscr{H}}=M(\varphi) .
$$

It is readily seen that $\mathscr{K}\left(t, x ; L_{\infty}, G\right)=\mathscr{K}\left(t, x ; L_{\infty}, L_{N}\right)$, for all $x \in G$. Therefore, for such $x$ the norm $\|x\|_{M(\varphi)}$ is equal to the norm $\|x\|_{Y}$, where $Y=\left(L_{\infty}, G\right)_{F}^{\mathscr{K}}$. On the other hand, for $x \in M(\varphi)$

$$
\frac{1}{t \log _{2}^{1 / 2}(2 / t)} \int_{0}^{t} x^{*}(s) d s \leq\|x\|_{M(\varphi)} \frac{\log _{2} \log _{2}(16 / t)}{\log _{2}^{1 / 2}(2 / t)} \longrightarrow 0 \text { as } t \rightarrow 0+.
$$

This implies that $M(\varphi) \subset G$ [10, page 156]. Thus $Y=M(\varphi)$, and (3.8) is proved. Equivalence (3.5) follows now, as already stated, from (3.7) and (3.8).

REMARK 3.5. Theorems 1.4 and 1.5 strengthen results of [18, 19], where similar assertions are obtained for sequence spaces $F$ satisfying more restrictive conditions. For instance, we can readily show that the norm of the dilation operator

$$
\sigma_{n} a=(\underbrace{a_{1}, \cdot, a_{1}}_{n}, \underbrace{a_{2}, \cdot, a_{2}}_{n}, \ldots)
$$

in the space $l_{1}(\ln )$ (see Example 3.6) is equal to $n$. Therefore, condition (11) from [19] fails for this space and the theorems obtained in [18, 19] cannot be applied to it. Similarly, the Marcinkiewicz space $M(\varphi)$ from Example 3.4 does not satisfy the conditions of Theorem 8 of [19].

Using Theorems 1.4 and 1.5, we can derive certain interpolation relations.

EXAMPLE 3.6. Let $\varphi \in \mathscr{P}$ and $1 \leq p<\infty$. Recall that the Lorentz space $\Lambda_{p}(\varphi)$ consists of all measurable functions $x=x(s)$ such that

$$
\|x\|_{\varphi, p}=\left\{\int_{0}^{1}\left(x^{*}(s)\right)^{p} d \varphi(s)\right\}^{1 / p}<\infty .
$$


In [19], V. A. Rodin and E. M. Semenov proved that

$$
\left\|\sum_{k=1}^{\infty} a_{k} r_{k}\right\|_{\varphi, p} \asymp\left\|\left(a_{k}\right)\right\|_{p},
$$

where $\varphi(s)=\log _{2}^{1-p}(2 / s)$ and $1<p<2$. Moreover, the space $\Lambda_{p}(\varphi)$ is the unique r.i.s. having this property. Note that $l_{p}=\left(l_{1}, l_{2}\right)_{\theta, p}$, where $\theta=2(p-1) / p$ [4, page 142]. Therefore, by Theorem 1.4, we obtain

$$
\left(L_{\infty}, G\right)_{\theta, p}=\Lambda_{p}(\varphi)
$$

for the same $p$ and $\theta$.

ACKnowledgement. The author is grateful to Prof. S. Montgomery-Smith for useful advices and to referees for their suggestions and remarks.

\section{REFERENCES}

[1] N. Asmar and S. Montgomery-Smith, On the distribution of Sidon series, Ark. Mat. 31 (1993), no. 1, 13-26. MR 94i:43006. Zbl 836.43011.

[2] S. V. Astashkin, K-monotone weighted couples generated by space noninvariant concerning to shift, Internat. Sem. Differential Equations (Samara), vol. 2, 1998, pp. 19-25.

[3] M. S. Baouendi and C. Goulaouic, Commutation de l'intersection et des foncteurs d'interpolation, C. R. Acad. Sci. Paris Sér. A-B 265 (1967), 313-315 (French). MR 37\#727. Zbl 166.10702.

[4] J. Bergh and J. Löfström, Interpolyatsionnye Prostranstva [Interpolation Spaces], Mir, Moscow, 1980 (Russian), Vvedenie (An introduction). Translated from English by V. S. Krjučkov and P. I. Lizorkin. MR 82c:46083.

[5] J. A. Brudnyl and N. J. Krugljak, Functors of real interpolation, Dokl. Akad. Nauk SSSR 256 (1981), no. 1, 14-17 (Russian). MR 82g:46115.

[6] _ Interpolation Functors and Interpolation Spaces. Vol. I, North-Holland Mathematical Library, vol. 47, North-Holland Publishing Co., Amsterdam, 1991, translated from Russian by Natalie Wadhwa with a preface by Jaak Peetre. MR 93b:46141. Zbl 743.46082.

[7] T. Holmstedt, Interpolation of quasi-normed spaces, Math. Scand. 26 (1970), 177-199. MR 54\#3440. Zbl 193.08801.

[8] J. Jakubowski and S. Kwapień, On multiplicative systems of functions, Bull. Acad. Polon. Sci. Sér. Sci. Math. 27 (1979), no. 9, 689-694. MR 82c:60014. Zbl 493.42036.

[9] S. V. Kislyakov and K. Shu, Real interpolation and singular integrals, Algebra i Analiz 8 (1996), no. 4, 75-109, translation in St. Petersburg Math. J. 8 (1997), no. 4, 593-615. MR 98c:46161.

[10] S. G. Kreĭn, J. I. Petunin, and E. M. Semënov, Interpolyatsiya Lineĭnykh Operatorov [Interpolation of Linear Operators], Nauka, Moscow, 1978 (Russian). MR 81f:46086. Zbl 499.46044.

[11] J. Lindenstrauss and L. Tzafriri, Classical Banach Spaces. II. Function spaces, Ergebnisse der Mathematik und ihrer Grenzgebiete [Results in Mathematics and Related Areas], vol. 97, Springer-Verlag, Berlin, 1979. MR 81c:46001. Zbl 403.46022.

[12] G. G. Lorentz, Relations between function spaces, Proc. Amer. Math. Soc. 12 (1961), 127132. MR 23\#A511. Zbl 124.31704.

[13] S. J. Montgomery-Smith, The distribution of Rademacher sums, Proc. Amer. Math. Soc. 109 (1990), no. 2, 517-522. MR 91a:60034. Zbl 696.60013.

[14] P. Nilsson, Reiteration theorems for real interpolation and approximation spaces, Ann. Mat. Pura Appl. (4) 132 (1982), 291-330. MR 86c:46089. Zbl 514.46049. 
[15] V. I. Ovchinnikov, The method of orbits in interpolation theory, Math. Rep. 1 (1984), no. 2, i-x and 349-515. MR 88d:46136. Zbl 875.46007.

[16] V. A. Rodin, Fourier series in symmetric space and interpolation of operators, Ph.D. thesis, Cand. Phys.-Math. Sci., Voronezh, 1973.

[17] V. A. Rodin and E. M. Semënov, The complementability of a subspace that is generated by the Rademacher system in a symmetric space, Funktsional. Anal. i Prilozhen. 13 (1979), no. 2, 91-92 (Russian). MR 80j:46048.

[18] V. A. Rodin and E. M. Semenov (eds.), Distribution functions of Rademacher series, Vseross School on Stoch. Methods, vol. 3, Tuapse, 1996.

[19] V. A. Rodin and E. M. Semyonov, Rademacher series in symmetric spaces, Anal. Math. 1 (1975), no. 3, 207-222. MR 52\#8905. Zbl 315.46031.

[20] G. Sparr, Interpolation of weighted $L_{p}$-spaces, Studia Math. 62 (1978), no. 3, 229-271. MR 80d:46055. Zbl 393.46029.

[21] H. Triebel, Teoriya Interpolyatsii, Funktional'nye Prostranstva, Differentisial'nye Operatory [Interpolation Theory. Function Spaces. Differential Operators], Mir, Moscow, 1980 (Russian), translated from English. Zbl 531.46025.

[22] R. Wallstén, Remarks on interpolation of subspaces, Function Spaces and Applications (Lund, 1986), Lecture Notes in Math., vol. 1302, Springer-Verlag, Berlin, 1988, pp. 410-419. MR 89h:46042. Zbl 662.46079.

[23] A. Zigmund, Trigonometricheskie Ryady. Tomy I [Trigonometric Series], Izdat. "Mir", Moscow, 1965 (Russian), translated from English by O. S. Ivasev Musatov. MR 31\#2554. Zbl 121.05804.

Sergey V. Astashin: Department of Mathematics, Samara Street University, ACADemic Pavlov STREet, 1, SAMARA, 443011, Russia

E-mail address: astashkn@ssu. samara.ru 


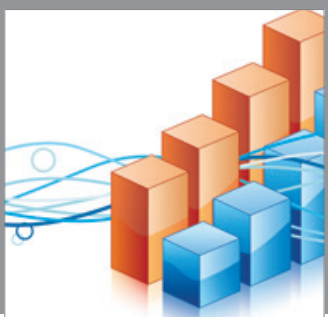

Advances in

Operations Research

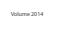

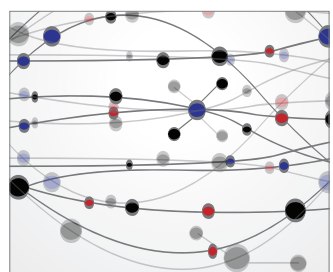

\section{The Scientific} World Journal
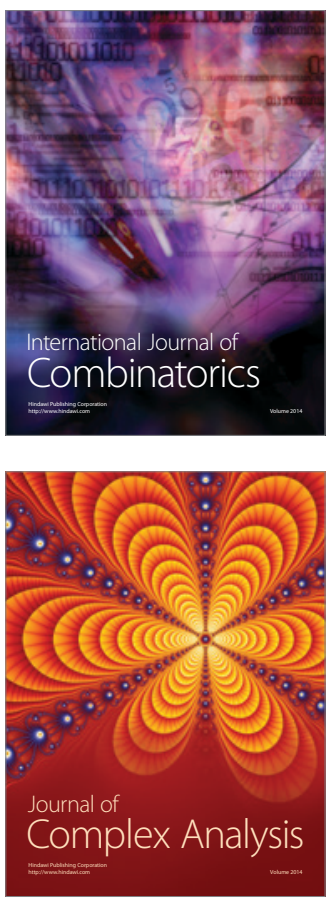

International Journal of

Mathematics and

Mathematical

Sciences
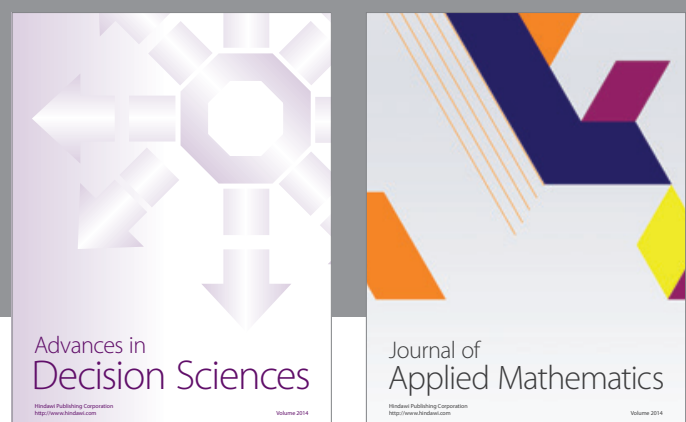

Journal of

Applied Mathematics
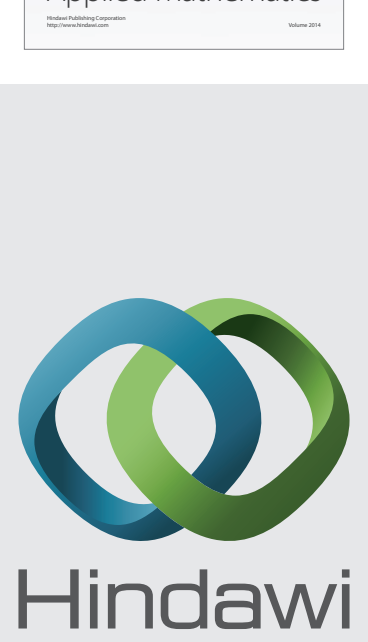

Submit your manuscripts at http://www.hindawi.com
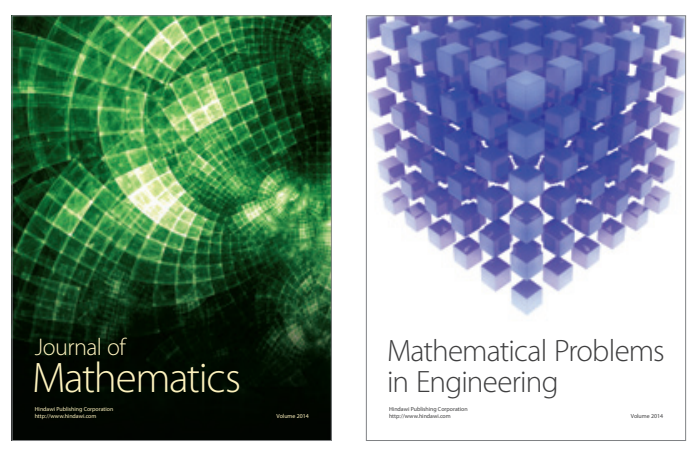

Mathematical Problems in Engineering
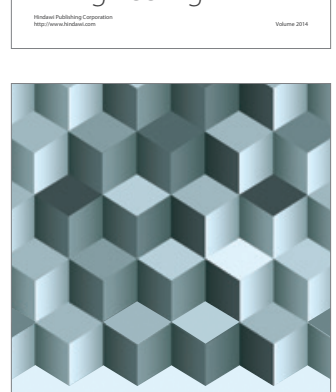

Journal of

Function Spaces
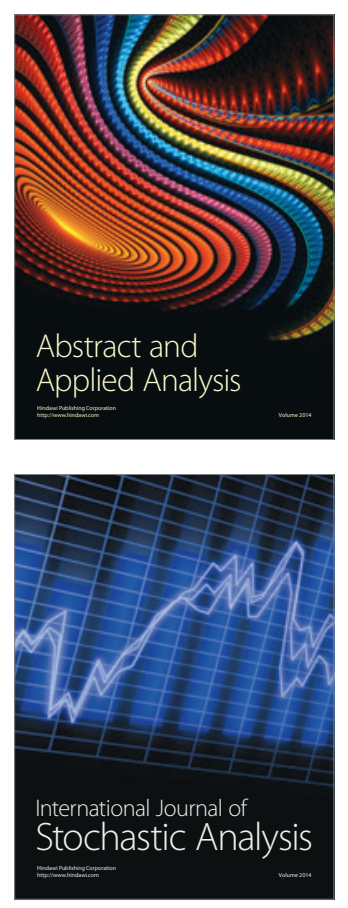

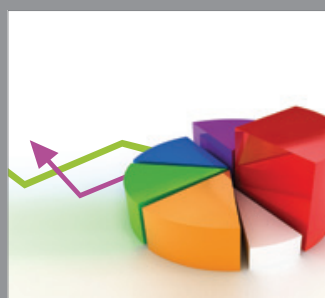

ournal of

Probability and Statistics

Promensencen
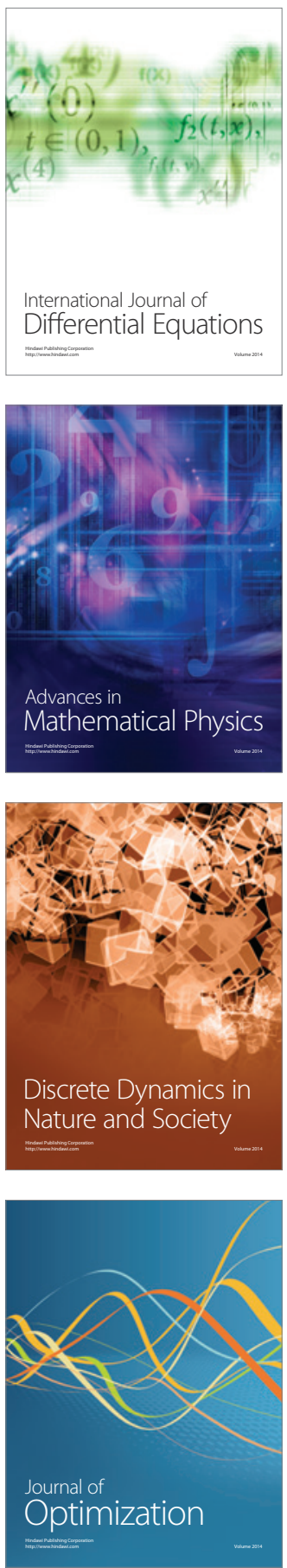